\title{
CYLD mutation characterizes a subset of HPV-positive head and neck squamous cell carcinomas with distinctive genomics and frequent cylindroma-like histologic features
}

\author{
Erik A. Williams $\mathbb{D}^{1} \cdot$ Meagan Montesion $\mathbb{1}^{1} \cdot$ Brian M. Alexander ${ }^{1} \cdot$ Shakti H. Ramkissoon ${ }^{1,2} \cdot$ Julia A. Elvin ${ }^{1} \cdot$ \\ Jeffrey S. Ross $\mathbb{D}^{1,3} \cdot$ Kevin Jon Williams $^{4} \cdot$ Krzysztof Glomski $^{5} \cdot$ Jacob R. Bledsoe $^{6} \cdot$ Julie Y. Tse $^{1} \cdot$ \\ Mark C. Mochel (D) $^{7}$
}

Received: 17 June 2020 / Revised: 20 August 2020 / Accepted: 20 August 2020 / Published online: 5 September 2020

(c) The Author(s) 2020. This article is published with open access

\begin{abstract}
Mutations in the tumor suppressor $C Y L D$, known to be causative of cylindromas, were recently described in a subset of highrisk (hr) HPV-positive head and neck squamous cell carcinomas (HNSCC). Pathologic and genetic characterization of these $C Y L D$-mutant carcinomas, however, remains limited. Here, we investigated whether $C Y L D$ mutations characterize a histopathologically and genomically distinct subset of hrHPV-positive HNSCC. Comprehensive genomic profiling via hybrid capture-based DNA sequencing was performed on 703 consecutive head and neck carcinomas with hrHPV sequences, identifying 148 unique cases $(21 \%)$ harboring CYLD mutations. Clinical data, pathology reports, and histopathology were reviewed. CYLD mutations included homozygous deletions $(n=61 / 148 ; 41 \%)$, truncations $(n=52$; $35 \%)$, missense $(n=26 ; 18 \%)$ and splice-site $(n=9 ; 6 \%)$ mutations, and in-frame deletion $(n=1 ; 1 \%)$. Among hrHPVpositive HNSCC, the $C Y L D$-mutant cohort showed substantially lower tumor mutational burden than $C Y L D$-wildtype cases $(n=555)$ (median 2.6 vs. $4.4 \mathrm{mut} / \mathrm{Mb}, p<0.00001)$ and less frequent alterations in PIK3CA (11\% vs. 34\%, $p<0.0001)$, $K M T 2 D(1 \%$ vs. $16 \%, p<0.0001)$, and $F B X W 7$ (3\% vs. $11 \%, p=0.0018)$. Male predominance $(94 \%$ vs. $87 \%)$, median age (58 vs. 60 years), and detection of HPV16 (95\% vs. 89\%) were similar. On available histopathology, 70\% of CYLD-mutant HNSCC (98/141 cases) contained hyalinized material, consistent with basement membrane inclusions, within crowded aggregates of tumor cells. Only $7 \%$ of $C Y L D$-wildtype cases demonstrated this distinctive pattern $(p<0.0001)$. Histopathologic patterns of $C Y L D$-mutant HNSCC lacking basement membrane inclusions included nonkeratinizing $(n=$ $22,16 \%$ ), predominantly nonkeratinizing (nonkeratinizing SCC with focal maturation; $n=10,7 \%)$, and keratinizing $(n=$ $11,8 \%)$ patterns. The latter two groups showed significantly higher frequency of PTEN alterations compared with other CYLD-mutant cases $(38 \%$ [8/21] vs. 7\% [8/120], $p=0.0004)$. Within our cohort of hrHPV-positive HNSCCs, CYLD mutations were frequent $(21 \%)$ and demonstrated distinctive clinical, histopathologic, and genomic features that may inform future study of prognosis and treatment.
\end{abstract}

These authors contributed equally: Julie Y. Tse, Mark C. Mochel

Supplementary information The online version of this article (https:// doi.org/10.1038/s41379-020-00672-y) contains supplementary material, which is available to authorized users.

Erik A. Williams

erwilliams@foundationmedicine.com

Extended author information available on the last page of the article

\section{Introduction}

The most recent World Health Organization classification of head and neck tumors divides squamous cell carcinoma (SCC) of the oropharynx into high-risk (hr) HPV-positive and hrHPV-negative tumors [1]. Among hrHPV-positive head and neck SCC (HNSCC), currently recognized morphologic variants include papillary, adenosquamous, lymphoepithelioma-like, and basaloid subtypes [1]. In addition to carcinomas of the oropharynx, hrHPV-positive SCC also occurs at a lower frequency in other head and neck sites including the sinonasal tract, oral cavity, and hypopharynx and larynx [2]. 
Nonkeratinizing histomorphology in SCC of the head and neck strongly correlates with HPV infection [3]. The basaloid subtype of oropharyngeal SCC, originally described by Wain et al. [4], contains a basaloid component of crowded cells with scant cytoplasm, often with thickened basement membranes and inclusions of basement membrane material, associated with foci of keratinization [4-6]. Some authors emphasize the "jigsaw-puzzle" pattern of closely apposed tumor lobules, cystic spaces with myxoid material, deposition of basement membrane material, and abrupt keratinization to delineate the basaloid variant of HNSCC from nonkeratinizing HPV-positive SCC, which often displays "basaloid" cytomorphology (i.e., tumor cells with scant cytoplasm and hyperchromatic nuclei) [7, 8]. Basaloid variant SCC with confirmed HPV positivity has shown a better prognosis than HPV-negative basaloid variant SCC [6, 9]. As such, some authorities emphasize HPV testing in SCCs with histologic features of the basaloid variant, to exclude the aggressive HPV-negative basaloid variant [10].

Molecular genetic profiles of HNSCC differ by HPV status $[11,12]$. The genes most commonly altered in HPVnegative HNSCC include TP53, CDKN2A, and CCDN1. In contrast, HPV-positive HNSCC demonstrates a low rate of TP53 and CDKN2A mutations, while PIK3CA mutations, $T R A F 3$ loss, and E2F1 amplifications are relatively common [11].

Mutations of $C Y L D$, which encodes an enzyme regulator of NF- $\mathrm{KB}$, have been previously characterized as causative of syndromic and sporadic cylindroma $[13,14]$ and were recently described in HNSCC [15]. A recent study demonstrated that HPV-positive HNSCC with mutations of either $C Y L D$ or TRAF3, which has similar function to $C Y L D$, demonstrated increased NF- $\mathrm{KB}$ activity, harbored HPV DNA in episomal form rather than integrated genomic form, and correlated with improved patient survival [15]. Histopathologic features and comprehensive genomic characteristics were not reported for this distinctive molecular subtype of HNSCC.

Given recent work by our group that correlated $C Y L D$ mutations in HPV-positive basaloid carcinomas of the anus with cylindroma-like histologic features, low tumor mutational burden (TMB), and low frequency of PIK3CA driver mutations [16], we hypothesized that $C Y L D$-mutant, HPVpositive HNSCCs may have similarly distinctive histologic and genomic features. In the current study, we retrospectively assessed HPV-positive head and neck carcinoma samples analyzed by comprehensive genomic profiling (CGP) for CYLD mutations. This analysis may enhance tumor categorization and inform further correlations with prognosis and potential therapeutic approaches to CYLDmutant tumors, such as recently investigated targeted inhibitors for cylindromatosis [17, 18].

\section{Materials and methods}

\section{Cohort and genomic analyses}

Approval for this study, including a waiver of informed consent and Health Insurance Portability and Accountability Act waiver of individual authorization, was obtained from the Western Institutional Review Board (Protocol No. 20152817). In the course of clinical care, physicians at outside institutions sent tumor samples for CGP by our Clinical Laboratory Improvement Amendments-certified, College of American Pathologists-accredited laboratory (Foundation Medicine, Inc., Cambridge, MA, USA). Here, we searched our database of cases with CGP to identify all HPV-positive head and neck carcinomas with CYLD mutation versus $C Y L D$-wildtype tumors. HPV status of each case was determined via the sequencing platform, as detailed below. We excluded adenocarcinomas and tumors of the salivary glands. In brief, board-certified pathologists confirmed pathologic diagnoses and tissue adequacy for all cases on routine hematoxylin and eosin (H\&E)-stained slides before DNA extraction. Macrodissection of sections was performed to achieve an estimated percent tumor nuclei (\% $\%$ TN) above $20 \%$ in each case, where $\% \mathrm{TN}$ is defined as 100 times the number of tumor cells divided by the total number of nucleated cells. For genomic assessment, $\geq 60 \mathrm{ng}$ of DNA was extracted from $40-\mu \mathrm{m}$ sections cut from formalin-fixed, paraffin-embedded tissue blocks containing tumor. Adaptor ligation hybrid capture, utilizing the Foundation One T7 baitset [19], was performed on the specimens. Supplementary Table 1 lists all sequenced genes in the T7 baitset. The Illumina HiSeq 4000 System was used to perform sequencing of captured libraries to a mean exon coverage depth of targeted regions of $>500 X$. Sequences were analyzed for genomic alterations, including short variant alterations (base substitutions, insertions, and deletions), copy number alterations (focal amplifications and homozygous deletions), and select gene fusions or rearrangements [19-21]. For maximum sensitivity and specificity of mutation detection in impure clinical specimens, sequencing was previously optimized and validated to detect base substitutions at a $\geq 5 \%$ mutant allele frequency, indels with a $\geq 10 \%$ mutant allele frequency with $\geq 99 \%$ accuracy, and fusions occurring within baited introns/exons with $>99 \%$ sensitivity [19]. TMB, quantified as mutations per $\mathrm{Mb}$, was assessed on $1.1 \mathrm{Mb}$ of sequenced DNA [21]. Up to 114 loci were assessed for determination of microsatellite instability (MSI) [22]. De novo assembly of nonhuman sequencing reads and comparison utilizing the basic local alignment search tool for nucleotides (BLASTn) against viral sequences in the comprehensive NCBI RefSeq database was used to detect HPV DNA sequences. HPV positivity was designated for cases with contigs $\geq 80$ 
nucleotides in length with $\geq 97 \%$ sequence identity to the Refseq sequence identified by BLASTn. Evaluated HPV types included HPV6, 11, 16, 18, 26, 31, 33, 35, 39, 40, 42, $43,44,45,51,52,53,54,55,56,58,59,61,62,64,66,67$, $68,69,70,71,72,73,81,82,83,84$, CP6108, and IS39. HPV16, 18, 33, and 58 were designated hr, and HPV6 was designated low-risk, as previously described [23].

\section{Mutational signatures}

The presence and quality of mutational signatures was evaluated for all samples with at least 20 nondriver missense mutations. Signatures were assigned through analysis of the trinucleotide context with profiling of the Sanger COSMIC cancer mutational signatures [24]. A positive signature required at least $40 \%$ of identified genomic alterations to fit to a known mutational process: APOBEC overexpression $(\mathrm{C}>\mathrm{T}$ and $\mathrm{C}>\mathrm{G}$ base substitutions), exposure to UV light $(\mathrm{C}>\mathrm{T}$ and $\mathrm{CC}>\mathrm{TT}$ base substitutions at dypirimidine sites), hypofunction of the BRCA tumor suppressor ( $>3$ base pair insertions or deletions with overlapping microhomology at breakpoint junctions), and defects in mismatch repair $(<3$ base pair insertions and deletions at mono/polynucleotide repeats) [24].

\section{Germline prediction}

Computational prediction of whether a particular CYLD mutation was germline was performed using a previously validated somatic-germline-zygosity algorithm [20]. This technique aligns sequencing reads and mutant allele frequencies for detected mutations and compares these to the expected values generated by the copy number model [19]. Based on this comparison, the algorithm calculates a prediction of whether the variant was germline, somatic, or ambiguous [20].

\section{Clinicopathologic analyses of head and neck carcinomas with CYLD mutations}

Outside institutional pathology reports that accompanied each specimen sent to Foundation Medicine for CGP were reviewed to collect clinicopathologic data, including patient age, gender, tumor site, and AJCC stage (8th edition) [25].

H\&E-stained sections from each available $C Y L D$-mutant hrHPV-positive carcinoma were assessed retrospectively by three pathologists (EAW, JYT, MCM) for histologic features, including growth pattern, deposition of basement membrane material, squamous differentiation, necrosis, and cytomorphology. The description of "basaloid" cytomorphology was applied to tumors composed of cells with scant cytoplasm and hyperchromatic nuclei. The designation of carcinomas with "cylindroma-like histopathologic features" was assigned principally by the presence of inclusions of hyalinized material, consistent with basement membrane inclusions reminiscent of those seen in cylindroma, within aggregates of carcinoma cells; however, additional histologic features, described in the "Results," were also common in this subgroup. The identification of keratinization was based on the histologic presence of squamous pearls or aggregates of cells with glassy, eosinophilic cytoplasm. SCCs lacking basement membrane inclusions were classified into one of three categories: "non-keratinizing" for SCCs lacking keratinization; "predominantly non-keratinizing" for SCCs in which keratinization was present but limited to small foci representing $<10 \%$ of the examined tumor area; and "keratinizing" for SCCs with keratinization in $\geq 10 \%$ tumor area. Histopathology of $C Y L D$-wildtype hrHPV-positive carcinomas was reviewed for comparison. Histopathologic assessment of all cases was limited to the sequenced tissue, representing one block of tumor per case. Reviewers were aware of the $C Y L D$ status when evaluating the histologic features. Although immunohistochemical slides were not available for retrospective review, we collected immunohistochemical findings from pathology reports.

Given the size of the cohort, Fisher's exact test was used for analyses of categorical data. For comparison of continuous parameters (age and TMB) between two groups, the non-parametric Mann-Whitney $U$ test was used. A twotailed $p$ value of $<0.05$ was considered statistically significant. For multiple simultaneous comparisons, the Bonferroni correction was applied.

\section{Results}

\section{Clinicopathologic features of CYLD-mutant hrHPV- positive head and neck carcinoma}

In our database, we identified 703 distinct hrHPV-positive head and neck carcinoma specimens, of which head and neck adenocarcinoma and cases of salivary gland origin were excluded as no HPV-positive CYLD-mutant cases were identified in these groups. In total, 148 distinct cases (21\%) featured $C Y L D$ mutations. Compared with $C Y L D$ wildtype head and neck carcinomas $(n=555), C Y L D$ mutant cases showed similar male predominance as CYLD-wildtype hrHPV-positive head and neck carcinoma ("CYLD-wildtype"; 94\% [139/148] vs. 87\% [484/555], respectively, not significant with corrected $p$ value; Table 1), as well as median age (58 vs. 60 years, NS; Table 1). The cohort of 148 CYLD-mutant carcinomas encompassed 40 primary HNSCC, 1 primary-site recurrence, and 107 metastatic samples. The metastatic samples were from the liver ( $n=35 / 107 ; 33 \%)$, lung $(n=30 ; 28 \%)$, 
Table 1 Comparative demographics, genomic alterations, and additional biomarkers of high-risk HPV-related head and neck carcinomas stratified by $C Y L D$ mutation status.

\begin{tabular}{|c|c|c|c|}
\hline & $C Y L D$-mutant & $C Y L D$-wildtype & $p^{*}$ \\
\hline Number of cases & 148 & 555 & \\
\hline$\%$ male & $94 \%(139 / 148)$ & $87 \%(484 / 555)$ & 0.02 \\
\hline Median age (range) & $58(38-89)$ & $60(8-89+)$ & 0.684 \\
\hline Cylindroma-like histopathology (\%) & $70 \%(98 / 141)$ & $7 \%(34 / 459)$ & $<0.0001$ \\
\hline Sequenced from liver metastasis (\%) & $24 \%(35 / 148)$ & $7 \%(37 / 555)$ & $<0.0001$ \\
\hline Sequenced from lung metastasis $(\%)$ & $20 \%(30 / 148)$ & $15 \%(83 / 555)$ & 0.131 \\
\hline Median TMB (Q1-Q3; mut/Mb) & $2.61(1.74-3.48)$ & $4.35(2.61-7.83)$ & $<0.0001$ \\
\hline HPV16 positive & $95 \%(140 / 148)$ & $89 \%(494 / 555)$ & 0.043 \\
\hline MSI high & $0 \%(0 / 138)$ & $<1 \%(2 / 513)$ & 1.000 \\
\hline \multicolumn{4}{|l|}{ PI3K/AKT/mTOR pathway } \\
\hline$P I K 3 C A$ & $11 \%$ & $34 \%$ & $<0.0001$ \\
\hline$K M T 2 D^{\mathrm{a}}$ & $1 \%$ & $16 \%$ & $<0.0001$ \\
\hline$F B X W 7$ & $3 \%$ & $11 \%$ & $\mathbf{0 . 0 0 1 8}$ \\
\hline PTEN & $12 \%$ & $13 \%$ & 0.679 \\
\hline $\operatorname{SOX} 2^{\mathrm{a}}$ & $2 \%$ & $6 \%$ & 0.085 \\
\hline STK11 & $2 \%$ & $4 \%$ & 0.442 \\
\hline \multicolumn{4}{|l|}{ Epigenetic regulation } \\
\hline$K M T 2 C$ & $5 \%$ & $11 \%$ & 0.037 \\
\hline EP300 & $5 \%$ & $6 \%$ & 0.840 \\
\hline \multicolumn{4}{|l|}{ DNA damage } \\
\hline TP53 & $4 \%$ & $11 \%$ & 0.008 \\
\hline$B C L 2 L 1$ & $12 \%$ & $5 \%$ & 0.009 \\
\hline \multicolumn{4}{|l|}{ Single pass transmembrane receptor } \\
\hline NOTCH1 & $6 \%$ & $8 \%$ & 0.598 \\
\hline \multicolumn{4}{|l|}{ Cell cycle regulation } \\
\hline$R B 1$ & $6 \%$ & $7 \%$ & 0.855 \\
\hline$C D K N 2 A$ & $1 \%$ & $6 \%$ & 0.019 \\
\hline CCND1 & $1 \%$ & $3 \%$ & 0.094 \\
\hline \multicolumn{4}{|l|}{ Receptor tyrosine kinase } \\
\hline FGFR3 & $1 \%$ & $7 \%$ & 0.004 \\
\hline$E R B B 2$ & $1 \%$ & $3 \%$ & 0.390 \\
\hline$E G F R$ & $0 \%$ & $2 \%$ & 0.081 \\
\hline \multicolumn{4}{|l|}{ Non-receptor tyrosine kinase } \\
\hline$S R C$ & $5 \%$ & $1 \%$ & 0.015 \\
\hline \multicolumn{4}{|l|}{ RAS/MAPK pathway } \\
\hline$K R A S$ & $1 \%$ & $2 \%$ & 0.320 \\
\hline
\end{tabular}

${ }^{\mathrm{a}}$ Limited data in the literature on role in PI3K/AKT signaling.

*The Bonferroni correction for 27 simultaneous comparisons was applied; rows with a significant $p$ value $(<0.0019)$ are highlighted in bold.

regional lymph nodes $(n=14 ; 13 \%)$, bone $(n=11 ; 10 \%)$, distant soft tissue $(n=9 ; 8 \%)$, distant lymph nodes $(n=4$; $4 \%)$, brain $(n=2 ; 2 \%)$, adrenal $(n=1 ; 1 \%)$, and small intestine $(n=1 ; 1 \%)$. Compared with HPV-related $C Y L D$ wildtype head and neck carcinoma, CYLD-mutant cases were sequenced much more frequently from liver samples (24\% [35/148] vs. $7 \%$ [37/555], $p<0.0001$; Table 1). Of the entire cohort of 148 CYLD-mutant carcinomas, known primary sites included oropharynx $(n=121$; including 51 at the base of tongue, 51 tonsillar, 18 oropharynx not further specified, and 1 soft palate), nasopharynx $(n=3)$, and sinonasal tract $(n=1$, which was an HPV16 positive case of sphenoid sinus origin). The remaining 23 cases were sequenced from metastatic samples, and the precise primary tumor site within the head and neck was not further specified. 


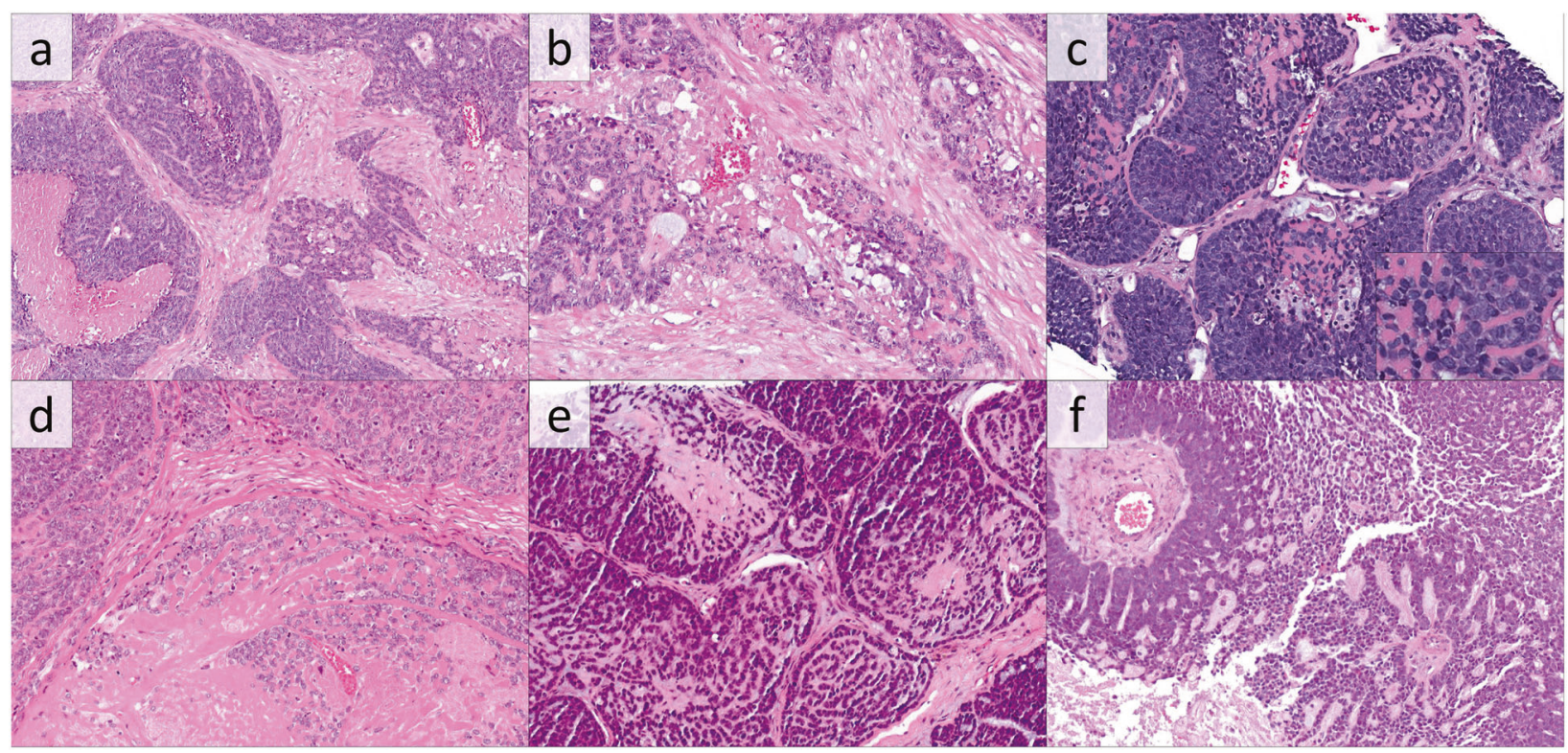

Fig. 1 CYLD-mutant hrHPV-positive squamous cell carcinomas of the head and neck with cylindroma-like histopathologic features. a, b Carcinoma, nonkeratinizing, with crowded aggregates of tumors cells with comedonecrosis and round inclusions of basement membrane material. Higher power examination (b) reveals focal mucinous stromal material associated with the hyaline inclusions (H\&E). (c and inset) Carcinoma composed of closely admixed and irregular tumor lobules with inclusions of basement membrane-like material and

The $C Y L D$-mutant carcinomas were clinically advanced. By AJCC 8th edition criteria [25], documented cases $(n=$ 141) were predominantly stage IV $(n=135 ; 96 \%)$, while the remaining documented cases were stage II $(n=5)$ and III $(n=1)$. Stage was unknown for seven cases.

HPV typing by CGP then BLASTn revealed that $C Y L D$ mutant and CYLD-wildtype HPV-positive carcinomas showed similar HPV16 predominance (95\% [140/148] vs. $89 \%$ [494/555], respectively, not significant with corrected $p$ value; Table 1). The remaining eight $C Y L D$-mutant cases contained HPV18 $(n=2), \operatorname{HPV} 33(n=4)$, or HPV35 $(n=$ 2) sequences. $C Y L D$-wildtype cases showed comparable rates of HPV18 (5.0\% [28/555], $p=0.064), \operatorname{HPV} 33$ (2.5\% [14/555], $p=1.0)$, and HPV35 (2.0\% [11/555], $p=1.0)$ sequences detected.

In comparison with HPV-related $C Y L D$-wildtype head and neck carcinoma, $C Y L D$-mutant cases were sequenced more frequently from liver samples $(24 \%$ [35/148] vs. $7 \%$ [37/ 555], $p<0.0001$ ) (Table 1). Sample frequency from other sites, such as lung, was not statistically different between the $C Y L D$-mutant and $C Y L D$-wildtype cohorts (Table 1).

\section{Histopathologic features of CYLD-mutant hrHPV- positive HNSCC}

Retrospective histopathologic review of 141 available $C Y L D$-mutant cases, consisting of representative sections of consisting of cells with scant cytoplasm and hyperchromatic nuclei (H\&E). d Carcinoma with prominent basement membrane inclusions and necrosis with somewhat paler chromatin than other examples (H\&E). e Carcinoma with closely apposed lobules with cribriform arrangement containing hyaline membrane and myxoid material (H\&E). f Rare pattern of CYLD-mutant, HPV-positive carcinoma with cribriform pattern, mucinous stromal material, and limited inclusions of basement membrane material (H\&E).

107 core or incisional biopsies and 34 excisional specimens, revealed SCCs with multiple patterns. Histopathologic patterns were grouped as follows: SCC with cylindromalike features, nonkeratinizing SCC, predominantly nonkeratinizing SCC, and keratinizing SCC.

The majority of CYLD-mutant SCCs (98 cases, 71\%) displayed cylindroma-like histologic features, defined principally by the presence of inclusions of basement membrane material within aggregates of tumor cells. All cases, by definition for this subgroup, contained inclusions of densely hyalinized material, typically globular, consistent with basement membrane inclusions, which were occasionally in continuity with surrounding thickened basement membranes (Fig. 1). These basement membrane inclusions were widely distributed in 52 cases (53\% of 98 ; Fig. 1a, b) and focal in 46 (47\%) cases (Fig. 1c). Tumor necrosis was present in 88 cases $(90 \%$; Fig. 1a, d-f). All cases were composed of lobules of crowded tumor cells with generally "basaloid" cytomorphology, with cells containing scant cytoplasm and atypical nuclei. In total, 86 cases (88\%) contained closely apposed tumor lobules. Foci of squamous differentiation, defined by keratin pearls or foci of aggregated squamous cells with dense eosinophilic cytoplasm, were present in 23 of these cases (23\% of 98; Fig. 2a). Squamous differentiation was focal in 19 cases and multifocal in 4 cases. Myxoid stroma (stromal mucinous material) was present in 52 cases (53\% of 98); in 36 cases, the 
Fig. 2 CYLD-mutant, hrHPVpositive squamous cell carcinomas of the head and neck with less common histologic patterns. a

Carcinoma showing both cylindroma-like inclusions of basement membrane material and squamous morules and pearls (H\&E). b Predominantly nonkeratinizing carcinoma with basaloid cytomorphology and limited foci of squamous differentiation (H\&E). c

Nonkeratinizing SCC (H\&E). d

Keratinizing squamous cell

carcinoma with glassy eosinophilic cytoplasm (H\&E).

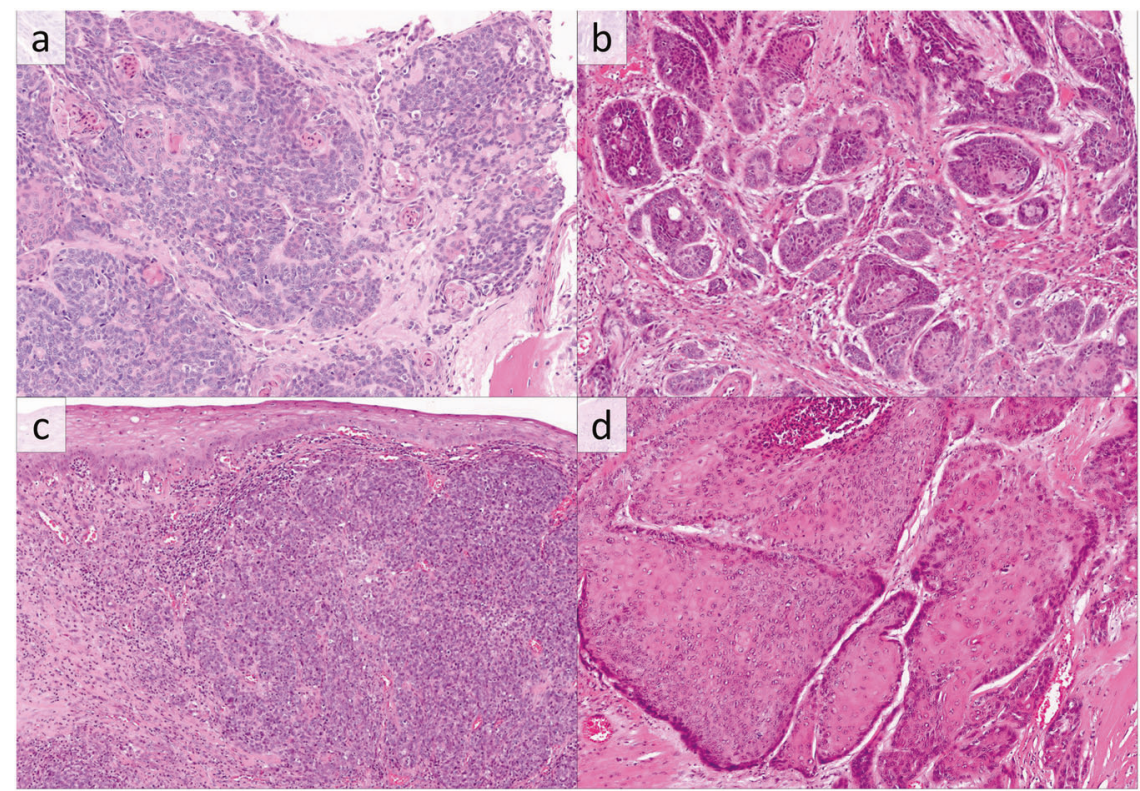

myxoid material was directly admixed with the epithelial proliferations, while the myxoid material was limited to peritumoral fibrotic stroma in 16 cases. When associated with tumor cells, the myxoid material tended to be either interstitial or mixed with hyaline inclusions; only one case showed discrete inclusions of myxoid material (Fig. 1f).

For comparison, histologic features of 459 available cases of $C Y L D$-wildtype, hrHPV-positive HNSCC were reviewed. Only 34 CYLD-wildtype HNSCC cases $(7 \%)$ contained inclusions of basement membrane material. These inclusions were widely distributed in only three cases and focal in the other 31. In the context of this cohort of HPVpositive HNSCC, basement membrane inclusions were significantly associated with CYLD mutation $(p<0.0001$; Table 1).

Of the 43 CYLD-mutant cases lacking basement membrane inclusions, histologic patterns were typical of HPVpositive oropharyngeal SCC [3], with patterns of nonkeratinizing SCC, predominantly nonkeratinizing SCC (nonkeratinizing SCC with focal maturation), and keratinizing SCC (Fig. 2b-d).

Twenty-two SCCs (16\%) were nonkeratinizing, lacking apparent keratinization on the tissue examined. Of these nonkeratinizing SCCs, 11 cases (50\%) showed necrosis, and deposition of myxoid material was present in 11 cases (50\%), which was limited to stroma in 2 cases.

Ten SCCs $(7 \%)$ were predominantly nonkeratinizing, with evidence of keratinization (squamous pearls or aggregates of cells with glassy eosinophilic cytoplasm) limited to small foci representing less than $10 \%$ of the examined tumor area. Seven of these cases $(70 \%)$ showed necrosis, while six cases contained myxoid material, limited to stroma in one case.
Eleven SCCs (8\%) were keratinizing with abundant squamous differentiation. Six cases showed necrosis, while three contained myxoid material, limited to stroma in two cases.

Review of immunohistochemical details in accompanying pathology reports found that the CYLD-mutant HNSCCs that had been evaluated by immunohistochemistry were consistently positive for p16 (99\%; 76/77 cases), p63 (100\%: 54/54 cases), p40 (100\%; 39/39 cases), and CK5/6 (93\%: 53/57 cases diffusely positive, 3/57 focal positive). CK7 was diffusely positive in only 2/21 CYLD-mutant cases, focally positive in $2 / 21$ cases, and negative in the remaining 17 cases. CYLD-mutant HNSCCs were consistently negative when stained for synaptophysin $(n=0 / 35$ cases), chromogranin ( $n=0 / 33)$, TTF1 $(n=0 / 23)$, CK20 $(n=0 / 15)$, and $\operatorname{CDX} 2(n=0 / 7)$.

\section{Comprehensive genomic profiling of CYLD-mutant hrHPV-positive HNSCC}

The set of CYLD mutations in our cohort consisted of homozygous deletions ( $n=61 / 148 ; 41 \%$ ), nonsense mutations $(n=29 ; 20 \%)$, truncating frameshifts $(n=18 ; 12 \%)$, truncating rearrangements $(n=5 ; 3 \%$; total truncating mutations $=52 / 148 ; 35 \%)$, missense mutations $(n=26$; $18 \%)$, splice-site mutations $(n=9 ; 6 \%)$, and in-frame deletions $(n=1 ; 1 \%)$ (Fig. 3a, b). One CYLD-mutant tumor had a missense mutation and a nonsense mutation (Fig. 3a) and is counted in each group.

Truncating mutations occurred in exons $4(n=1), 5(n=$ 8), $6(n=1), 8(n=1), 9(n=7), 10(n=2), 12(n=6), 13$ $(n=3), 14(n=1), 15(n=1), 16(n=7), 17(n=3), 18$ $(n=4), 19(n=2)$, and $20(n=5)$. Missense mutations 
a
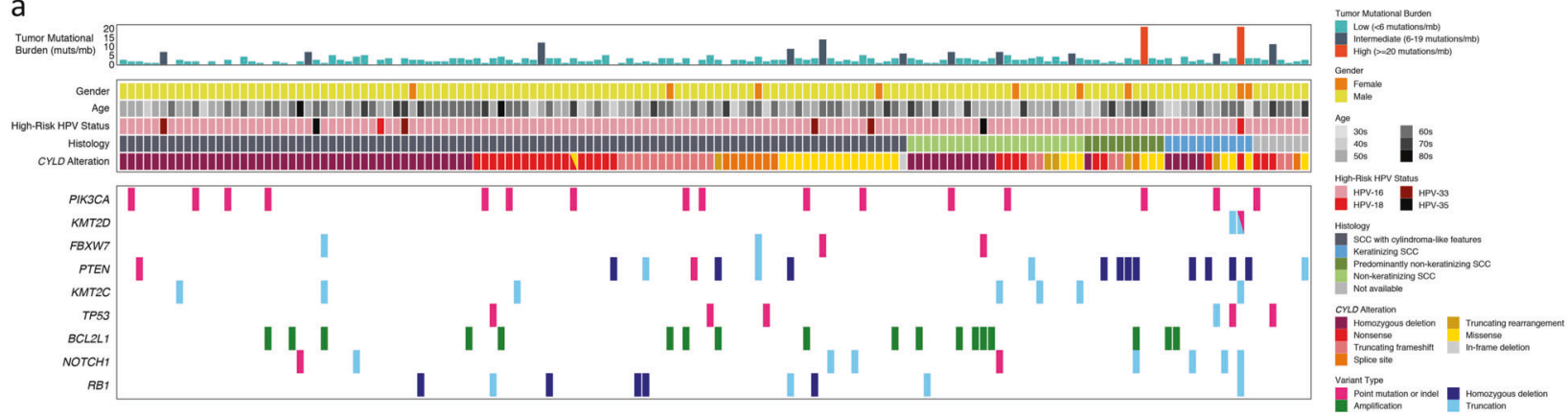

b

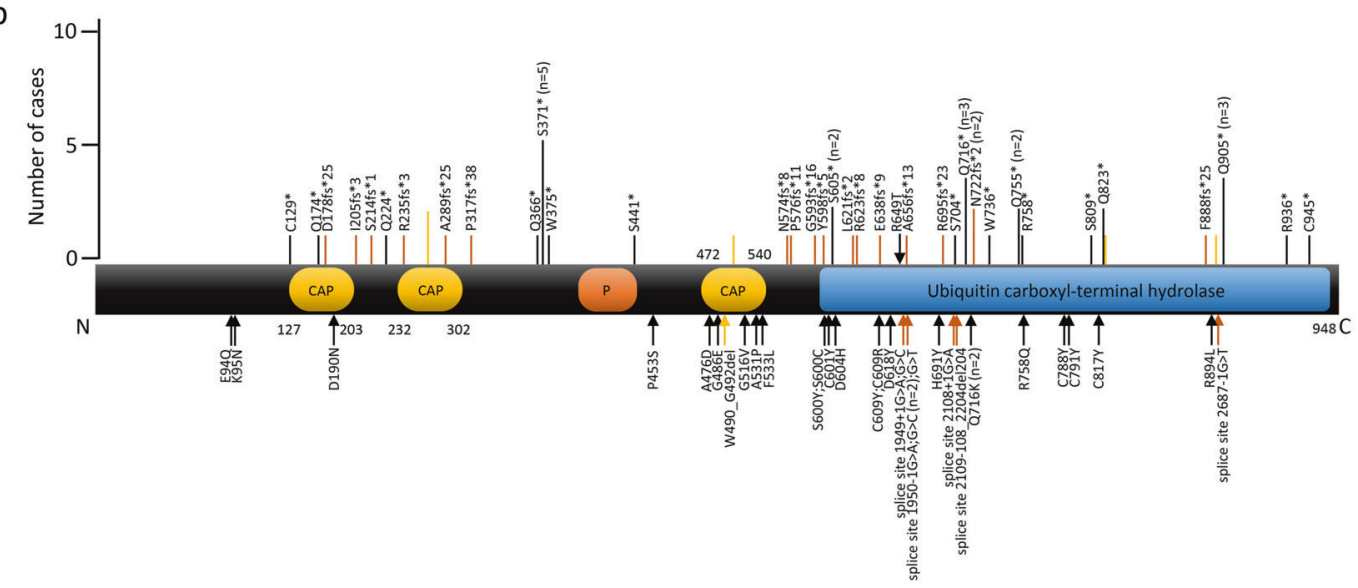

Fig. 3 Clinicopathologic features and molecular landscape of HPV-positive $C Y L D$-mutant head and neck squamous cell carcinoma. a Summary of clinical features, histopathology, and molecular alterations in $C Y L D$-mutant head and neck carcinoma. b Schematic of functional domains of $C Y L D$ (transcript NM_015247), to include identified mutation sites. $C Y L D$ nonsense mutations $(n=29)$, frameshift mutations $(n=18)$, and truncating rearrangement site $(n=5)$ are

occurred in exons $4(n=2), 5(n=1), 10(n=3), 11(n=$ 3), $12(n=4), 13(n=4), 15(n=1), 16(n=2), 17(n=1)$, $18(n=3)$, and $19(n=1)$.

Figure $3 \mathrm{a}$ and Table 1 show the most frequent genomic alterations outside the $C Y L D$ locus in $C Y L D$-mutant versus $C Y L D$-wildtype HNSCC tumors. The $C Y L D$-mutant group showed substantially lower TMB and less frequent alterations in PIK3CA, KTM2D, and FBXW7, all three of which are involved in the PI3K-AKT-mTOR pathway (Table 1). Alterations in $\mathrm{PTCHI}$ and $\mathrm{SMO}$ were rare or absent from the $C Y L D$-mutant (1\% and $0 \%$, respectively) and $C Y L D$ wildtype (1\% and $0 \%$ ) groups.

$C Y L D$-mutant cases with predominantly nonkeratinizing SCC (SCC with focal maturation) $(n=10)$ and keratinizing SCC $(n=11)$ patterns showed significantly higher frequency of PTEN alterations compared with other $C Y L D$-mutant cases, with four PTEN-mutant cases in each histopathologic pattern $(38 \%$ [8/21] vs. $7 \%$ [8/120], $p=$ 0.0004; dark green and blue versus dark gray and light green histology tiles in Fig. 3a). These cases also exhibited a higher frequency of alterations in the adjacent $F A S$ gene denoted by black, orange, and yellow bars, respectively (top of diagram; length of bar number of cases). Missense mutations $(n=25)$, splice-site mutations $(n=9)$, and in-frame deletions $(n=1)$ are labeled with black, orange, and yellow arrows, respectively (predominantly lower diagram). In total, 61 cases with homozygous deletions in $C Y L D$ are not shown. CAP cytoskeleton-associated proteins; P phosphorylation region.

(24\% [5/21] vs. $3 \%$ [3/120], $p=0.0019)$. Demographics and site of sequenced specimen (primary vs. metastases) were similar between cases with each histologic pattern. We found no significant genetic differences between $C Y L D$-mutant carcinomas with cylindroma-like features $(n=98)$ versus $C Y L D$-mutant nonkeratinizing $\operatorname{SCC}(n=$ 22) (dark gray versus light green histology tiles in Fig. 3a). No $C Y L D$-mutant or $C Y L D$-wildtype cases demonstrated the NFIB-MYB gene fusion typical of adenoid cystic carcinoma.

Patients with $C Y L D$-mutant tumors sequenced from primary $(n=41)$ vs. metastatic sites $(n=107)$ showed similar age and sex. Cases sequenced from metastatic sites showed a significantly elevated rate of BCL2L1 amplification compared with primary-site tumor samples (16\% [17/107] vs. $2 \%[1 / 41], p=0.0248)$, particularly in cases sequenced from liver metastases (20\%; 7/35 cases). Median TMB was not significantly different between primary-site versus metastatic tumor samples (1.7 vs. $2.6 \mathrm{mut} / \mathrm{Mb}, p=0.072$ ). No identifiable genetic differences were seen between specific primary anatomic origins of HNSCC (oropharynx, 
nasopharynx, sinonasal tract), but the number of nonoropharyngeal cases was limited.

Six $C Y L D$-mutant cases underwent analysis for mutational signatures. Five of these cases were identified with an APOBEC signature. Of the available $C Y L D$-wildtype cases, 208 underwent mutational signature analysis and showed a similar frequency of the APOBEC signature $(63 \%, n=131$ / 208).

Comparison of cases with $C Y L D$ mutations within the ubiquitin hydrolase domain (exons 12-20) versus outside the protease domain (exons 4-11) revealed no significant clinical, histologic, or genomic differences. Within our CYLD-mutant cohort, cases with HPV16 sequences showed no significant differences when compared with cases carrying other hrHPV reads (HPV18, HPV33, or HPV35), although numbers in this latter group were limited.

Twenty of the $148 C Y L D$-mutant cases had available SGZ algorithm data to predict germline status, and all 20 $C Y L D$ mutations were predicted to be somatic.

Comparison of CYLD-wildtype HNSCC with cylindroma-like inclusions vs. those without these inclusions revealed that cases with cylindroma-like features showed significantly lower TMB (median 2.6 vs. 4.4 mut/ $\mathrm{Mb}, p=0.0219)$, and a lower frequency of PIK3CA (12\% [4/34] vs. $35 \%$ [150/425], $p=0.0042), K M T 2 D(3 \%$ [1/34] vs. $18 \%$ [76/425], $p=0.0284)$, and $F B X W 7$ genomic alterations $(0 \%$ [0/34] vs. $13 \%$ [53/425], $p=0.0229)$. In these parameters, CYLD-wildtype HNSCC with cylindroma-like histologic features resembled CYLDmutant HNSCC, which often showed cylindroma-like features. In addition, $C Y L D$-wildtype HNSCC with cylindroma-like histology showed a higher frequency of NFKBIA truncating alterations $(12 \%$ [4/34] vs. $1 \%$ [4/425], $p=0.0014)$. No other significant differences between $C Y L D$-wildtype HNSCC with vs. without cylindroma-like features were identified.

\section{Discussion}

Here, we report that the presence of $C Y L D$ mutation defines a relatively frequent subset of HPV-related HNSCC that exhibits histopathologic features reminiscent of cylindroma and distinctive genomics. Our group recently demonstrated an analogous subset of HPV-positive, CYLD-mutant basaloid anal carcinomas with cylindroma-like histologic features [16]. In addition to similar histology, the HPV-positive $C Y L D$-mutant anal carcinoma and HPV-positive CYLDmutant head and neck carcinoma share unusually low TMB, low frequency of PIK3CA mutation, and a possible predilection for liver metastases.

Our current findings in HPV-positive HNSCCs correlate and may unify previously reported genetic and histopathologic findings for the $C Y L D$-mutant subtype of HNSCC. A recent detailed genetic study using TCGA data found that one-third of HPV-positive SCCs of the head and neck contained mutually exclusive mutations of either $C Y L D$ or TRAF3, each of which is normally an endogenous inhibitor of NF-kB. Thus, these mutations were found to upregulate NF-kB activity [15]. HPV-positive SCCs with these mutations demonstrated several additional distinctive features, including HPV DNA in episomal form, rather than chromosomally integrated, and high levels of mRNAs associated with motility and proliferation. These $C Y L D$ - and TRAF3-mutant cases also showed improved clinical survival compared with HPV-positive HNSCC tumors lacking mutations in these genes. TMB was not reported. Status of the PIK3CA gene was examined in $36 \mathrm{HPV}$-positive HNSCC tumors (4 CYLD-mutant, 9 TRAF3-mutant, 23 CYLD-wildtype/TRAF3-wildtype), and PIK3CA mutations tended to be mutually exclusive from $C Y L D / T R A F 3$ mutations. While this detailed study did not report histopathology, our series correlates HPV-positive, CYLD-mutant SCCs in this anatomic location with cylindroma-like histologic features, particularly the presence of basement membrane inclusions.

Earlier mRNA expression profiling and cluster analysis [26], as well as RNA sequencing studies [27], identified two distinct subtypes of HPV-positive HNSCC characterized by differential mRNA expression, copy number alterations, HPV DNA integration into host chromosomes, and frequency of PIK3CA mutations. One subtype (termed HPVclassical or HPV-keratinocyte, HPV-KRT) was characterized by expression of genes in keratinocyte differentiation, high levels of host chromosomal integration of HPV DNA, gain of chromosome 3q, frequent PIK3CA mutations, and keratinization on histopathology. The other subtype (termed HPV-immune response, HPV-IMU) was characterized by expression of genes implicated in immune responses and mesenchymal differentiation, low frequency of host chromosomal integration of HPV DNA, infrequent PIK3CA mutations, loss of chromosome 16q, and infrequent keratinization [26, 27]. Interestingly, $C Y L D$, which was listed in the supplemental data of reference [27] along with a large number of other genes on chromosome 16q, showed somewhat decreased expression in the HPV-IMU group [27]. Correlating these prior studies [15, 26, 27] with our current findings, we note that our cohort of HPV-positive, CYLD-mutant HNSCCs shares some key features with the HPV-IMU subtype of HNSCC. In particular, we found that $P I K 3 C A$ mutations are significantly less frequent in $C Y L D$ mutant than in $C Y L D$-wildtype HPV-positive HNSCC, and that the majority of these cases demonstrate histopathologic features of crowded tumor cell aggregates with basement membrane inclusions and limited keratinization (Fig. 1). In addition, as noted above, Hajek et al. showed that $C Y L D$ - 
mutant HNSCC generally lacked chromosomally integrated HPV DNA [15], another feature of the HPV-IMU subtype of HNSCC [26, 27].

From a morphologic standpoint, many cases in our cohort of HPV-positive, CYLD-mutant HNSCC display histopathologic features of the basaloid variant of HNSCC [4-6], suggesting that CYLD mutation may be a marker of HPV-positive basaloid variant SCC. Basaloid SCC is composed of crowded aggregates of cells with scant cytoplasm and with associated glassy eosinophilic material surrounding tumor lobules and entrapped as small round inclusions, variably termed "hyalinosis" or "hyaline stromal cores." The jigsaw puzzle-like pattern of closely situated irregular lobules of tumor cells and the presence of hyaline material deposition have been emphasized as features that distinguish the basaloid variant of SCC from the more common nonkeratinizing HPV-associated HNSCC $[6,8,28]$. In a classic study of ten basaloid SCCs of the tongue, hypopharynx, and larynx, Wain et al. defined basaloid SCC as an admixture of basaloid and squamous foci: basaloid areas consisted of densely arranged small cells with scant cytoplasm showing lobular growth, small cystic spaces containing myxoid material, and collections of hyaline material, while squamous zones manifested as either surface SCC in situ or an invasive component with typical keratinizing features such as keratin pearls and prominent intercellular bridges [4]. Some cases displayed a cribriform pattern of epithelial cells with matrix material likened to adenoid cystic carcinoma. By electron microscopy, cystic spaces contained either loose granules or replicated basement membrane material. In a later study of 40 similar cases, Banks et al. identified the hyaline material surrounding and within tumor lobules in approximately two thirds of cases and further noted the frequent presence of comedonecrosis [5]. Comparative study of oropharyngeal and laryngeal/hypopharyngeal basaloid SCCs has shown similar histologic features, including deposits of hyaline material and necrosis [29]. To the authors' knowledge, CYLD status has not been reported previously for histopathologically well-characterized subtypes of HNSCC [4-6, 29, 30].

The association of histopathologic features of the basaloid variant of SCC, particularly hyaline deposition, with CYLD mutation suggests a novel correlation between genotype and histologic phenotype in HNSCC. Our impression, especially in light of $C Y L D$ mutation, the fundamental alteration in familial and sporadic cylindromas [13, 14], is that the deposition of this hyalinized basement membrane material most resembles that seen in cylindroma. To the authors' knowledge, study of genomic alterations within the basaloid variant of HNSCC is limited to a 2004 study of HNSCC subtypes, including seven basaloid carcinomas, using microsatellite markers which found that basaloid SCC tended to show loss of heterozygosity at loci on $9 \mathrm{p}$ and $11 \mathrm{q}$ [30]. Interestingly, CYLD-wildtype carcinomas with cylindroma-like basement membrane inclusions showed strikingly similar genomics to $C Y L D$-mutant cases, with similarly low TMB and significantly less frequent alterations in $P I K 3 C A, K M T 2 D$, and $F B X W 7$. Furthermore, these cases were enriched for inactivating alterations in $N F$ kappa-B inhibitor alpha (NFKBIA), leading us to consider that cylindroma-like features in HNSCC may be associated with non-CYLD mutations that activate related pathways. These potential mechanisms of histogenesis, including $T R A F 3$, which was not sequenced in this study, require further investigation.

Studies of the prognosis of basaloid SCC of the head and neck have found variable results, ranging from worse to improved prognosis for the basaloid subtype [31-34]. These studies are complicated, however, by the variable presence of HPV infection, which is common in the oropharynx and which plays an important role in prognosis of HNSCC $[6,9]$. Furthermore, some of these prognostic studies did not clearly delineate nonkeratinizing oropharyngeal SCC, sometimes called "basaloid" SCC in earlier studies, from the bona fide basaloid variant of SCC.

With respect to HPV infection among basaloid SCCs of the head and neck, Begum et al. found that $76 \%$ of basaloid SCCs occurring in the oropharynx were HPV-positive while nonoropharyngeal cases (hypopharynx, larynx, oral, and sinonasal) were rarely positive for HPV [9]. Chernock et al. similarly demonstrated consistent HPV positivity by in situ hybridization among basaloid SCCs occurring in the oropharynx, while laryngeal and hypopharyngeal cases were consistently negative [6]. Both studies found that HPVpositive cases have improved prognosis compared to HPVnegative cases [6,9]. The finding by Hajek et al. [15] that HPV-positive, CYLD-mutant HNSCCs demonstrate a more favorable prognosis than $C Y L D$-wild-type cases suggests that $C Y L D$ mutation, or potentially the surrogate of correlated histopathologic features, may inform the clinical phenotype of HPV-positive HNSCC.

CYLD, a de-ubiquitinating enzyme, suppresses both the $\mathrm{NF}-\kappa \mathrm{B}$ pathway $[35,36]$ and the $\mathrm{c}$-Jun N-terminal kinase pathway [37-39]. Experimental models have shown that CYLD loss promotes aggressive behavior in various malignancies, including cutaneous SCC [40], pancreatic carcinoma [41], and hepatocellular carcinoma [39]. CYLD mutation has been most extensively studied in the context of cutaneous cylindromas in both syndromic manifestations, such as Brooke-Spiegler syndrome, and sporadic tumors [13]. A variety of mutations of CYLD have been characterized, typically resulting in truncation of the encoded enzyme [14, 42-44].

Morphologically similar lesions, such as adenoid cystic carcinoma or salivary basal cell adenocarcinoma, may arise 
in the oral cavity and oropharynx [45, 46], necessitating awareness of oropharyngeal SCC variants, including those with cylindroma-like features and $C Y L D$ mutation. The histopathologic presence of necrosis, mitotic activity, and nuclear pleomorphism favors basaloid variant SCC, and by analogy the cylindroma-like carcinoma described here, over adenoid cystic carcinoma [47].

Basal cell adenocarcinoma, which usually arises in the parotid gland but may develop in minor salivary glands, shares with the $C Y L D$-mutant HNSCCs in this study several features including basaloid cellular composition, increased basement membrane material with inclusions, and, as suggested here, $C Y L D$ mutation. Both tumor types also display peripheral palisading of nuclei and foci of necrosis [48]. A recent study identified $C Y L D$ mutations within both basal cell adenomas and basal cell adenocarcinomas, perhaps accounting for some of the morphologic overlap with the cases in this current study [49]. Another study found CTNNB1 mutations in basal cell adenomas and, among other alterations, focal $C Y L D$ deletion and biallelic inactivation of NFKBIA in a case of basal cell adenocarcinoma [50]. Although few cases of HPV testing of basal cell adenocarcinoma have been reported, rare tested cases have been HPV-negative [51], contrasting the HNSCC cases in this study. Furthermore, basaloid SCCs are generally negative for CK7 and CD117, aiding in the distinction of basal cell adenocarcinoma of the salivary gland [47].

By immunohistochemistry, basaloid variant SCC of the head and neck is p63 positive and usually positive for keratins, albeit sometimes focally [52]. Tumors are negative for synaptophysin and chromogranin, which can be useful for distinguishing basaloid SCC from small cell carcinoma $[10,52]$. The positivity for p63 and negativity for neuroendocrine markers also distinguishes basaloid SCC from the rare entity of HPV-associated neuroendocrine carcinoma of the oropharynx, which has the opposite immunophenotype [53, 54]. Interestingly, a recent study of basaloid SCC showed that a majority of tumors were positive for SOX10 [55], a feature shared with most cylindromas $[56,57]$, as well as some many other cutaneous adnexal tumors and salivary gland tumors [58].

Cylindrocarcinomas of the skin are extremely rare and, ultimately, dissimilar to basaloid HNSCC. While both are carcinomas related to $C Y L D$ alterations, cylindrocarcinoma consists of a malignant proliferation associated with a precursor cylindroma [59]. Although CYLD mutations have been shown to be present in sporadic and syndromic cylindromas [13], molecular characterization of cylindrocarcinomas, beyond their occasional association with syndromes of germline $C Y L D$ mutation, is limited to characterizations of TP53 alterations $[60,61]$.

In our assessment, basaloid carcinoma of the anus is closely analogous to the CYLD-mutant oropharyngeal carcinomas described in this study. In a 2005 report of basaloid carcinomas of the anus, Chetty et al. noted that the histologic features of basaloid cytomorphology, comedonecrosis, and basement membrane inclusions were shared by both the basaloid anal carcinomas and those occurring in the oropharynx [62]. In addition to these overlapping histopathologic changes, we further note that these carcinomas from both sites show consistent $C Y L D$ mutations, HPV positivity, low TMB, low frequency of PIK3CA mutations, and likely propensity to metastasize to the liver [16].

Limitations of our study primarily concern the nature of our cases, which were drawn from a database of aggressive and advanced stage (AJCC 8th edition) cancers sent for analysis to search for potentially targetable genetic alterations. Thus, indolent tumors or those cured by excision were highly unlikely to be included. Follow-up data were also limited, and thus future studies will be needed assess the clinical course of these cancers, including prognostic features and therapeutic responses. TRAF3 mutation and HPV genomic integration were not evaluated in this study. In addition, we were able to review only the histopathology of the sequenced tissue, representing one block of tumor, most often that of a core or incisional biopsy, potentially limiting the ability to detect focal histologic features. Because the initial histologic review focused on the CYLD-mutant tumors to establish the range of histologic features, thereby prompting a later comparative review of $C Y L D$-wildtype cases, CYLD mutational status was known at the time of review, presenting the possibility of observer bias. Finally, immunohistochemistry was not available for evaluation beyond review of details from outside pathology reports.

In summary, we found that over one in five HPV-positive head and neck carcinomas in our cohort carry CYLD mutations, and that the presence of $C Y L D$ mutations defines a subset of HNSCC tumors that often display cylindromalike histopathologic features and exhibit unusually low TMB and infrequent alterations in PIK3CA. Characterization of HPV-positive, CYLD-mutant carcinoma may assist in classification of HNSCC and potentially inform prognosis and experimental therapies for the patients affected $[17,18]$, both topics that will require additional study.

\section{Compliance with ethical standards}

Conflict of interest EAW, MM, BA, JAE, JSR, SHR, and JYT are employees of Foundation Medicine, Inc., a wholly owned subsidiary of Roche Holdings, Inc. and Roche Finance Ltd, and these employees have equity interest in an affiliate of these Roche entities. KJW receives research funding from Novo Nordisk for studies unrelated to cancer. KG, JRB, and MCM declare no conflict of interest.

IRB approval IRB approval status: reviewed and approved by Western IRB; Protocol No. 20152817. 
Publisher's note Springer Nature remains neutral with regard to jurisdictional claims in published maps and institutional affiliations.

Open Access This article is licensed under a Creative Commons Attribution 4.0 International License, which permits use, sharing, adaptation, distribution and reproduction in any medium or format, as long as you give appropriate credit to the original author(s) and the source, provide a link to the Creative Commons license, and indicate if changes were made. The images or other third party material in this article are included in the article's Creative Commons license, unless indicated otherwise in a credit line to the material. If material is not included in the article's Creative Commons license and your intended use is not permitted by statutory regulation or exceeds the permitted use, you will need to obtain permission directly from the copyright holder. To view a copy of this license, visit http://creativecommons. org/licenses/by/4.0/.

\section{References}

1. Westra W, Boy S, El-Mofty S, Gillison M, Schwartz M, Syrjanen $\mathrm{S}$, et al. Tumours of the oropharynx (base of tongue, tonsils, adenoids): Squamous cell carcinoma, HPV-positive. In: El-Naggar A, Chan J, Grandis J, Takata T, Slootweg P, editors. WHO classification of head and neck tumours. 4th ed. Lyon, France: International Agency for Research on Cancer; 2017. p. 136-8.

2. El-Naggar AK, Chan JKC, Grandis JR, Takata T, Slootweg PJ, editors. WHO classification of head and neck tumours. 4th ed. Lyon, France: International Agency for Research on Cancer; 2017.

3. Chernock RD. Morphologic features of conventional squamous cell carcinoma of the oropharynx: 'Keratinizing' and 'Nonkeratinizing' histologic types as the basis for a consistent classification system. Head Neck Pathol. 2012;6:41-7.

4. Wain SL, Kier R, Vollmer RT, Bossen EH. Basaloid-squamous carcinoma of the tongue, hypopharynx, and larynx: report of 10 cases. Hum Pathol. 1986;17:1158-66.

5. Banks ER, Frierson HF, Mills SE, George E, Zarbo RJ, Swanson PE. Basaloid squamous cell carcinoma of the head and neck. A clinicopathologic and immunohistochemical study of 40 cases. Am J Surg Pathol. 1992;16:939-46.

6. Chernock RD, Lewis JS, Zhang Q, El-Mofty SK. Human papillomavirus-positive basaloid squamous cell carcinomas of the upper aerodigestive tract: a distinct clinicopathologic and molecular subtype of basaloid squamous cell carcinoma. Hum Pathol. 2010;41:1016-23.

7. El-Mofty SK. HPV-related squamous cell carcinoma variants in the head and neck. Head Neck Pathol. 2012;6:55-62.

8. Udager AM, McHugh JB. Human papillomavirus-associated neoplasms of the head and neck. Surg Pathol Clin. 2017;10:35-55.

9. Begum S, Westra WH. Basaloid squamous cell carcinoma of the head and neck is a mixed variant that can be further resolved by HPV status. Am J Surg Pathol. 2008;32:1044-50.

10. Bishop JA, Sciubba JJ, Westra WH. Squamous cell carcinoma of the oral cavity and oropharynx. Surg Pathol Clin. 2011;4:1127-51.

11. Lawrence MS, Sougnez C, Lichtenstein L, Cibulskis K, Lander E, Gabriel SB, et al. Comprehensive genomic characterization of head and neck squamous cell carcinomas. Nature. 2015;517:576-82.

12. Leemans CR, Snijders PJF, Brakenhoff RH. The molecular landscape of head and neck cancer. Nat Rev Cancer. 2018;18:269-82.

13. Rashid M, van der Horst M, Mentzel T, Butera F, Ferreira I, Pance A, et al. ALPK1 hotspot mutation as a driver of human spiradenoma and spiradenocarcinoma. Nat Commun. 2019;10:1-10.

14. Bignell GR, Warren W, Seal S, Takahashi M, Rapley E, Barfoot $\mathrm{R}$, et al. Identification of the familial cylindromatosis tumoursuppressor gene. Nat Genet. 2000;25:160-5.
15. Hajek M, Sewell A, Kaech S, Burtness B, Yarbrough WG, Issaeva N. TRAF3/CYLD mutations identify a distinct subset of human papillomavirus-associated head and neck squamous cell carcinoma. Cancer. 2017;123:1778-90.

16. Williams EA, Montesion M, Sharaf R, Corines J, Patel PJ, Gillespie BJ, et al. CYLD-mutant cylindroma-like basaloid carcinoma of the anus: a genetically and morphologically distinct class of HPV-related anal carcinoma. Mod Pathol. 2020. https://doi.org/ 10.1038/s41379-020-0584-2.

17. Danilenko M, Stamp E, Stocken DD, Husain A, Zangarini M, Cranston A, et al. Targeting tropomyosin receptor kinase in cutaneous CYLD defective tumors with pegcantratinib the TRAC randomized clinical trial. JAMA Dermatol. 2018;154:913-21.

18. Rajan N, Elliott R, Clewes O, MacKay A, Reis-Filho JS, Burn J, et al. Dysregulated TRK signalling is a therapeutic target in CYLD defective tumours. Oncogene. 2011;30:4243-60.

19. Frampton GM, Fichtenholtz A, Otto GA, Wang K, Downing SR, He J, et al. Development and validation of a clinical cancer genomic profiling test based on massively parallel DNA sequencing. Nat Biotechnol. 2013;31:1023-31.

20. Sun JX, He Y, Sanford E, Montesion M, Frampton GM, Vignot S, et al. A computational approach to distinguish somatic vs. germline origin of genomic alterations from deep sequencing of cancer specimens without a matched normal. PLoS Pathog. 2018;14:1-13.

21. Chalmers ZR, Connelly CF, Fabrizio D, Gay L, Ali SM, Ennis R, et al. Analysis of 100,000 human cancer genomes reveals the landscape of tumor mutational burden. Genome Med. 2017;9: $1-14$.

22. Trabucco SE, Gowen K, Maund SL, Sanford E, Fabrizio DA, Hall $\mathrm{MJ}$, et al. A novel next-generation sequencing approach to detecting microsatellite instability and pan-tumor characterization of 1000 microsatellite instability-high cases in 67,000 patient samples. J Mol Diagn. 2019;21:1053-66.

23. Muñoz N, Bosch FX, De Sanjosé S, Herrero R, Castellsagué X, Shah KV, et al. Epidemiologic classification of human papillomavirus types associated with cervical cancer. N Engl J Med. 2003;348:518-27.

24. Zehir A, Benayed R, Shah RH, Syed A, Middha S, Kim HR, et al. Mutational landscape of metastatic cancer revealed from prospective clinical sequencing of 10,000 patients. Nat Med. 2017;23:703-13

25. Amin MB, Edge SB, Greene FL, Byrd DR, Brookland RK, Washington MK, et al. AJCC cancer staging system. 8th ed. New York: Spinger; 2017.

26. Keck MK, Zuo Z, Khattri A, Stricker TP, Brown CD, Imanguli M, et al. Integrative analysis of head and neck cancer identifies two biologically distinct HPV and three non-HPV subtypes. Clin Cancer Res. 2015;21:870-81.

27. Zhang Y, Koneva LA, Virani S, Arthur AE, Virani A, Hall PB, et al. Subtypes of HPV-positive head and neck cancers are associated with HPV characteristics, copy number alterations, PIK3CA mutation, and pathway signatures. Clin Cancer Res. 2016;22:4735-45.

28. Lewis JS, Chernock RD. Human papillomavirus and epstein barr virus in head and neck carcinomas: suggestions for the new WHO classification. Head Neck Pathol. 2014;8:50-8.

29. Ereño C, Gaafar A, Garmendia M, Etxezarraga C, Bilbao FJ, López JI. Basaloid squamous cell carcinoma of the head and neck. Head Neck Pathol. 2008;2:83-91.

30. Choi HR, Roberts DB, Johnigan RH, Sturgis EM, Rosenthal DI, Weber RS, et al. Molecular and clinicopathologic comparisons of head and neck squamous carcinoma variants: common and distinctive features of biological significance. Am J Surg Pathol. 2004;28:1299-310. 
31. Soriano E, Faure C, Lantuejoul S, Reyt E, Bolla M, Brambilla E, et al. Course and prognosis of basaloid squamous cell carcinoma of the head and neck: a case-control study of 62 patients. Eur $\mathbf{J}$ Cancer. 2008;44:244-50.

32. Fritsch VA, Gerry DR, Lentsch EJ. Basaloid squamous cell carcinoma of the oral cavity: an analysis of 92 cases. Laryngoscope. 2014;124:1573-8.

33. Fritsch VA, Lentsch EJ. Basaloid squamous cell carcinoma of the oropharynx: an analysis of 650 cases. Otolaryngol Head Neck Surg. 2013;148:611-8

34. Fritsch VA, Lentsch EJ, Carolina S. Basaloid squamous cell carcinoma of the head and neck: location means everything. J Surg Oncol. 2014;109:616-22.

35. Ikeda F, Dikic I. CYLD in ubiquitin signaling and tumor pathogenesis. Cell. 2006;125:643-5.

36. Massoumi R, Chmielarska K, Hennecke K, Pfeifer A, Fässler R. Cyld inhibits tumor cell proliferation by blocking Bcl-3-dependent NF-кB signaling. Cell. 2006;125:665-77.

37. Ke H, Augustine CK, Gandham VD, Jin JY, Tyler DS, Akiyama $\mathrm{SK}$, et al. CYLD inhibits melanoma growth and progression through suppression of the jnk/ap-1 and $\beta 1$-integrin signaling pathways. J Investig Dermatol. 2013;133:221-9.

38. Sato Y, Goto E, Shibata Y, Kubota Y, Yamagata A, Goto-Ito S, et al. Structures of CYLD USP with Met1-or Lys63-linked diubiquitin reveal mechanisms for dual specificity. Nat Struct Mol Biol. 2015;22:222-9.

39. Nikolaou K, Tsagaratou A, Eftychi C, Kollias G, Mosialos G, Talianidis I. Inactivation of the deubiquitinase CYLD in hepatocytes causes apoptosis, inflammation, fibrosis, and cancer. Cancer Cell. 2012;21:738-50.

40. Alameda JP, Moreno-Maldonado R, Navarro M, Bravo A, Ramírez A, Page A, et al. An inactivating CYLD mutation promotes skin tumor progression by conferring enhanced proliferative, survival and angiogenic properties to epidermal cancer cells. Oncogene. 2010;29:6522-32.

41. Xie S, Wu Y, Hao H, Li J, Guo S, Xie W, et al. CYLD deficiency promotes pancreatic cancer development by causing mitotic defects. J Cell Physiol. 2019;234:9723-32.

42. Almeida S, Maillard C, Itin P, Hohl D, Huber M. Five new CYLD mutations in skin appendage tumors and evidence that aspartic acid 681 in CYLD is essential for deubiquitinase activity. J Investig Dermatol. 2008;128:587-93.

43. Nasti S, Pastorino L, Bruno W, Gargiulo S, Battistuzzi L, Zavattaro E, et al. Five novel germline function-impairing mutations of CYLD in Italian patients with multiple cylindromas. Clin Genet. 2009;76:481-5.

44. Nagy N, Farkas K, Kemény L, Széll M. Phenotype-genotype correlations for clinical variants caused by CYLD mutations. Eur J Med Genet. 2015;58:271-8.

45. Wilson TC, Robinson RA. Basal cell adenocarcinoma and basal cell adenoma of the salivary glands: a clinicopathological review of seventy tumors with comparison of morphologic features and growth control indices. Head Neck Pathol. 2015;9:205-13.

46. Hay AJ, Migliacci J, Karassawa Zanoni D, McGill M, Patel S, Ganly I. Minor salivary gland tumors of the head and neckmemorial Sloan Kettering experience: Incidence and outcomes by site and histological type. Cancer. 2019;125:3354-66.

47. Mills AM, Stelow EB, Mills SE. A basaloid squamous cell carcinoma of the supraglottic larynx. Pathol Case Rev. 2011;16:167-72.
48. Andreadis D, Nomikos A, Epivatianos A, Poulopoulos A, Barbatis C. Basaloid squamous cell carcinoma versus basal cell adenocarcinoma of the oral cavity. Pathology. 2005;37:560-3.

49. Rito M, Mitani Y, Bell D, Mariano FV, Almalki ST, Pytynia KB, et al. Frequent and differential mutations of the CYLD gene in basal cell salivary neoplasms: linkage to tumor development and progression. Mod Pathol. 2018;31:1064-72.

50. Jo VY, Sholl LM, Krane JF. Distinctive patterns of CTNNB1 ( $\beta$-catenin) alterations in salivary gland basal cell adenoma and basal cell adenocarcinoma. Am J Surg Pathol. 2016;40:1143-50.

51. Haeggblom L, Ursu RG, Mirzaie L, Attoff T, Gahm C, Nordenvall $\mathrm{LH}$, et al. No evidence for human papillomavirus having a causal role in salivary gland tumors. Diagn Pathol. 2018;13:44.

52. Shah AA, Jeffus SK, Stelow EB. Squamous cell carcinoma variants of the upper aerodigestive tract: a comprehensive review with a focus on genetic alterations. Arch Pathol Lab Med. 2014;138:731-44.

53. Kraft S, Faquin WC, Krane JF. HPV-associated neuroendocrine carcinoma of the oropharynx: a rare new entity with potentially aggressive clinical behavior. Am J Surg Pathol. 2012;36: 321-30.

54. Jo VY, Krane JF, Pantanowitz L, Monaco SE. HPV-associated neuroendocrine carcinomas of the head and neck in FNA biopsies: clinicopathologic features of a rare entity. Cancer Cytopathol. 2019;127:26-34.

55. Rooper LM, Mccuiston AM, Westra WH, Bishop JA. SOX10 immunoexpression in basaloid squamous cell carcinomas: a diagnostic pitfall for ruling out salivary differentiation. Head Neck Pathol. 2019;13:543-7.

56. Cassarino DS, Su A, Robbins BA, Altree-Tacha D, Ra S. SOX10 immunohistochemistry in sweat ductal/glandular neoplasms. J Cutan Pathol. 2017;44:544-7.

57. Evangelista MTP, North JP. MYB, CD117 and SOX-10 expression in cutaneous adnexal tumors. J Cutan Pathol. 2017;44: 444-50.

58. Hsieh MS, Lee YH, Chang YL. SOX10-positive salivary gland tumors: a growing list, including mammary analogue secretory carcinoma of the salivary gland, sialoblastoma, low-grade salivary duct carcinoma, basal cell adenoma/adenocarcinoma, and a subgroup of mucoepidermoid carcinoma. Hum Pathol. 2016;56: $134-42$.

59. Kazakov DV, Zelger B, Rütten A, Vazmitel M, Spagnolo DV, Kacerovska D, et al. Morphologic diversity of malignant neoplasms arising in preexisting spiradenoma, cylindroma, and spiradenocylindroma based on the study of 24 cases, sporadic or occurring in the setting of brooke-spiegler syndrome. Am J Surg Pathol. 2009;33:705-19.

60. Kazakov DV, Grossmann P, Spagnolo DV, Vanecek T, Vazmitel M, Kacerovska D, et al. Expression of p53 and TP53 mutational analysis in malignant neoplasms arising in preexisting spiradenoma, cylindroma, and spiradenocylindroma, sporadic or associated with brooke-spiegler syndrome. Am J Dermatopathol. 2010;32:215-21.

61. Biernat W, Peraud A, Wozniak L, Ohgaki H. P53 mutations in sweat gland carcinomas. Int J Cancer. 1998;76:317-20.

62. Chetty R, Serra S, Hsieh E. Basaloid squamous carcinoma of the anal canal with an adenoid cystic pattern: histologic and immunohistochemical reappraisal of an unusual variant. Am J Surg Pathol. 2005;29:1668-72. 


\section{Affiliations}

Erik A. Williams $\mathbb{( 1 )}^{1} \cdot$ Meagan Montesion $\mathbb{1}^{1} \cdot$ Brian M. Alexander $^{1} \cdot$ Shakti H. Ramkissoon ${ }^{1,2} \cdot$ Julia A. Elvin $^{1} \cdot$ Jeffrey S. Ross $\mathbb{D}^{1,3} \cdot$ Kevin Jon Williams $^{4} \cdot \mathrm{Krzysztof} \mathrm{Glomski}^{5} \cdot$ Jacob R. Bledsoe $\mathbb{D}^{6} \cdot$ Julie Y. Tse $^{1}$ •

Mark C. Mochel $\mathbb{D}^{7}$

1 Foundation Medicine, Inc., 150 Second Street, Cambridge, MA 02141, USA

2 Wake Forest Comprehensive Cancer Center and Department of Pathology, Wake Forest School of Medicine, Winston-Salem, NC 27157, USA

3 Department of Pathology, State University of New York Upstate Medical University, 766 Irving Avenue, Syracuse, NY 13210, USA

4 Department of Physiology, Department of Medicine, Lewis Katz School of Medicine at Temple University, Philadelphia, PA 19140, USA
5 Hartford Pathology Associates, 80 Seymour Street, Hartford, CT 06102, USA

6 Department of Pathology, University of Massachusetts Medical School, One Innovation Drive, Worcester, MA 01605, USA

7 Departments of Pathology and Dermatology, Virginia Commonwealth University School of Medicine, 1200 East Marshall Street, Richmond, VA 23298, USA 